\title{
Rams Infertility and Sperms Mitochondrial Genome Defect
}

\author{
S. A. Hatif \\ A..S..Abood \\ Coll. of Vet Med/ Univ. of Baghdad
}

\begin{abstract}
This study was included a collection of 24 semen samples from healthy rams in Baghdad province, performed in Baghdad- college of veterinary medicine .Sperms stained with florescent dye ( ethidium bromide ) and subjected to florescent microscopical examination used by UV- light to visualized the defect in the mitochondrial sheeth covered the mid piece. The results gives the abnormalities of mitochondrial mid piece ( $\mathrm{mt}$ sheeth), the sperms appeared in 6 types of defect, included mixed defect in the same sample. The defect included $5(20.8 \%)$ cases interrupted distribution of $\mathrm{mt}$ genome, $4(16.6 \%)$ narrow, $10(41.6 \%)$ thickness , 8 (33.3\%)irregular , 7(29.16\%) short and 2(8.3\%) absent of mid piece. The aim of this study, determination the defect of mitochondrial sheeth of mid piece, in activity, motility, low quality of sperm and the role of infertility in ram .
\end{abstract}

\section{Introduction}

Many studies were concentration on sperms defect and infertility .Sperm quality is more important than quantity, sperm cells that are unable to move a common problem or a shaped abnormally, cannot reach an oocyte, with too little ATP to supply energy, sperm cannot move effectively, fertility declines (1) Sperm metabolism under aerobic (O2) and anaerobic condition, and consumption of nonorganic and organic constituents are Inoitols , Sorbitol ,Mannitol, erythritol, glycerol and Glycerylphosphorylcholine (2). Mammalian spermatozoa expend energy, generated as intracellular ATP, largely on motility mitochondrial oxidative phosphorylation, for which oxygen is friend, and glycolysis, for which sugar is friend, can provide the energy (3). The typical animal cell contains hundreds of mitochondria that produce the cells ATP through oxidative phosphorelation and regulate multiple cellular processes ,the

\section{Materials and methods}

Collection of 24 ram semen samples from healthy rams in Baghdad province, performed in Baghdad- college of veterinary medicine via aspiration from tail of epididymis, using needle gauge 21 . Sperms stained with ethidium bromide dye in order to stain mitochondrial sheath mitochondrial genome is critical for normal cellular energy (4) Mature mammalian sperm are known to contain 22-75 mitochondria, which form a tight helix around the flagellar basis of the midpiece, providing the ATP necessary for flagellar propulsion (5) In mammalian cells ,mitochondria and the nucleus are the only organelles that possess DNA (6) . Each mitochondrion is estimated to contain 2-10 mtDNA copies (7) There is increasing evidence that mitochondrial DNA (mtDNA) anomalies in sperm may lead to infertility (8) Because the sheath of mitochondria represented the mitochondrial DNA and genome.Therefore this study included

1-staind mid piece of sperm by ethidium bromide.

2- microscopical examination using UV light and without UV light .

3-Detectio the mitochondrial Sheeth (mtDNA) defect in sperm .

coverd mid piece, dye prepared by solution $0.25 \mathrm{gm}$ from ethidium bromide in $50 \mathrm{ml}$ Deestel Water to get in final concentrate $5 \mathrm{mg} / \mathrm{ml}$ (9).All samples subjected to microscopical examination , with UV light detection . 


\section{Results}

We are utilized 24 semen samples of rams were collected from tail of epididymis. Motility test refered Low motility of sperms (20\% - 40\%). The results referred the variation defect of mid piece when stained with ethidium bromide to obvious mitochondrial genome (mt DNA ) which represented the mitochondrial sheeth surrounded by plasma membrane of mid piece all

samples subjected to microscopical examination . the results gives 6 abnormal cases included, mixed defect in the sheeth of mitochondria Table 1 . The defect include 5 ( 20.8\%) cases interrupted distribution of mt genome Fig1, 4 (16.6\%) narrow Fig 6-7 , 10 (41.6\%) thickness Fig $3,8(33.3 \%)$ irregular Fig 2-5, $7(29.16 \%)$ short Fig 8 and 2(8.3\%) absent of mid piece Fig4 .
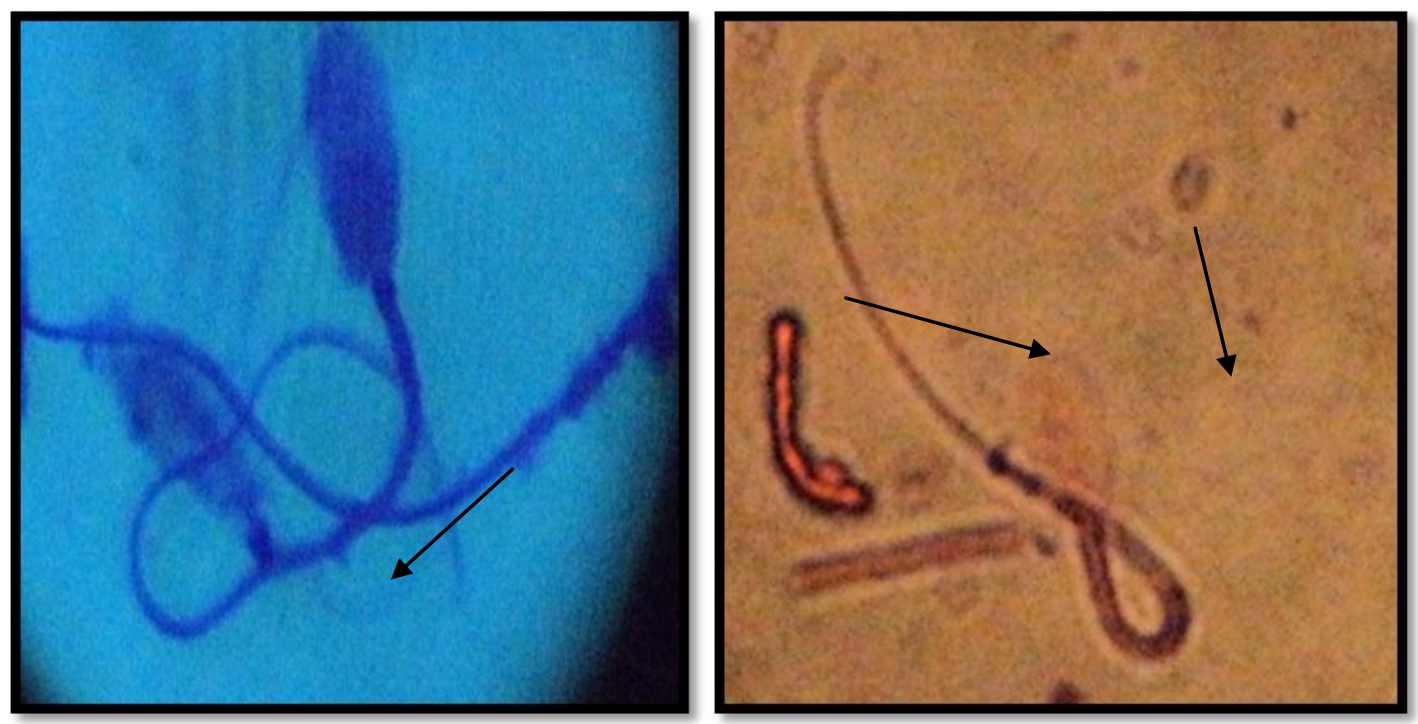

Fig: 1- interrupted mid piece .UV light( oil immersion) Fig:2 - irregular and normal mid piece. UV(O.I)
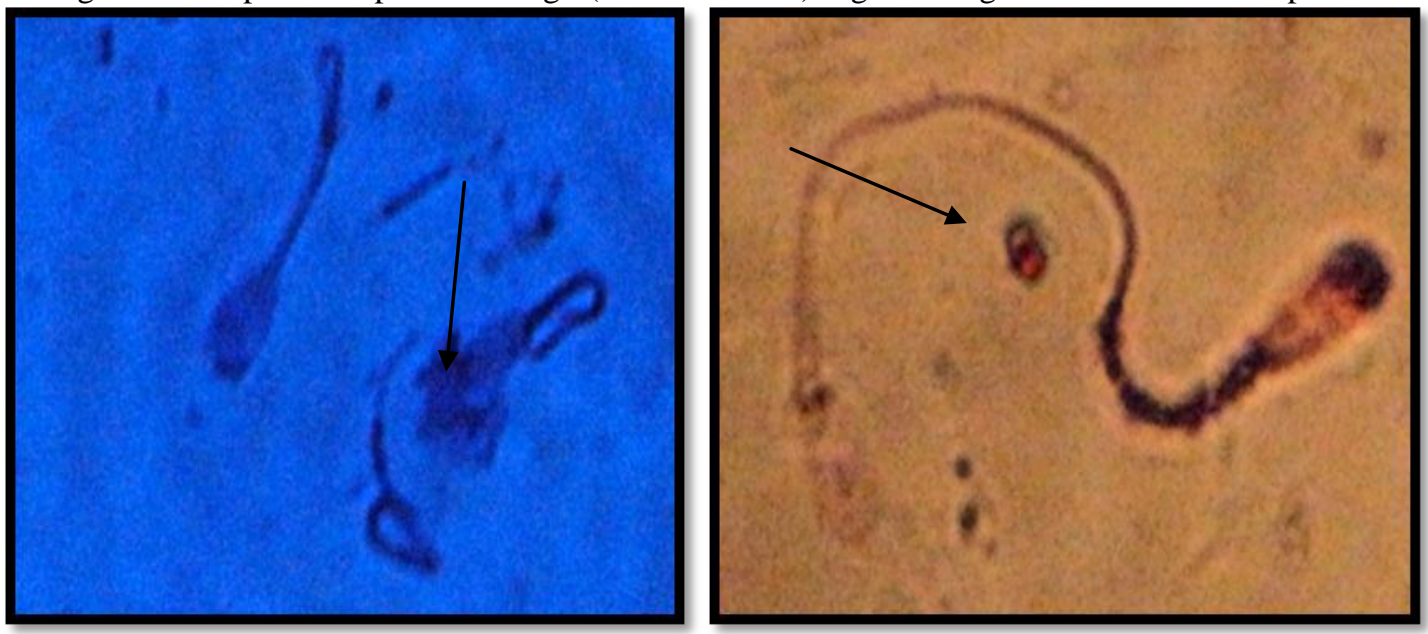

Fig:3 thickness and irregular mid piece(O.I)

Fig:4 absent mid piece. UV light (O.I) 

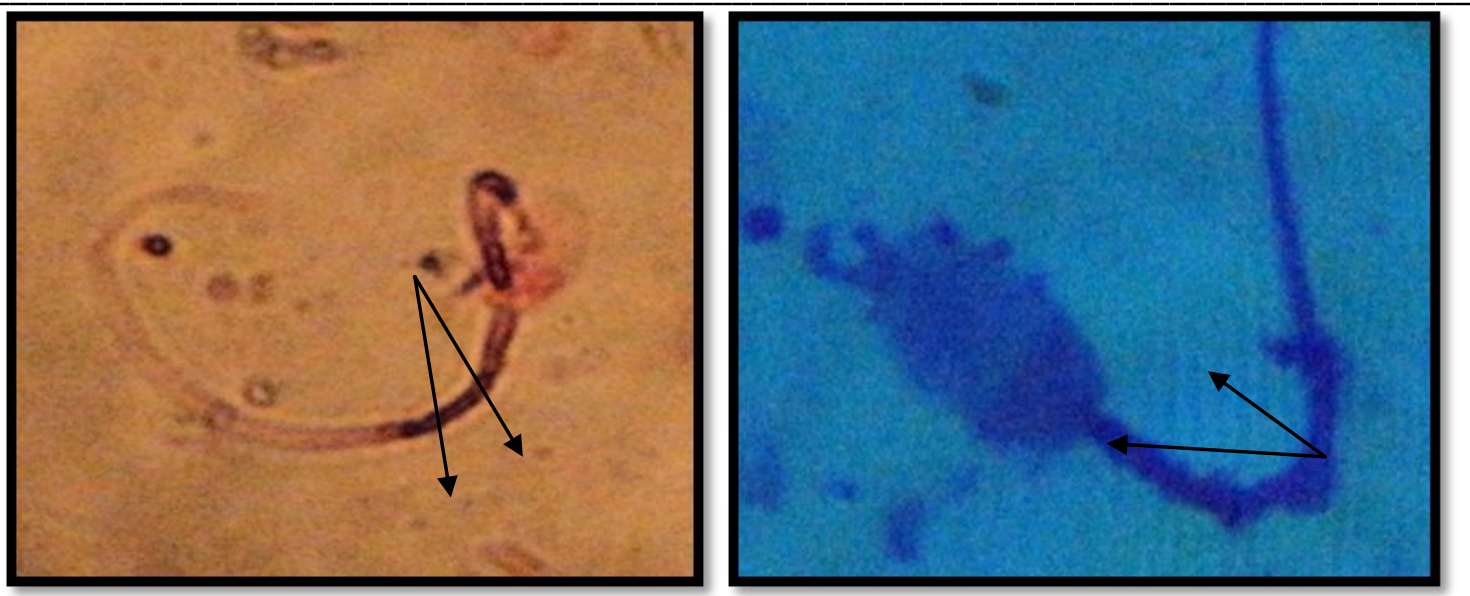

Fig: 5 irregular with mid piece. UV light(O.I)

Fig: 6 narrow mid piece(O.I)

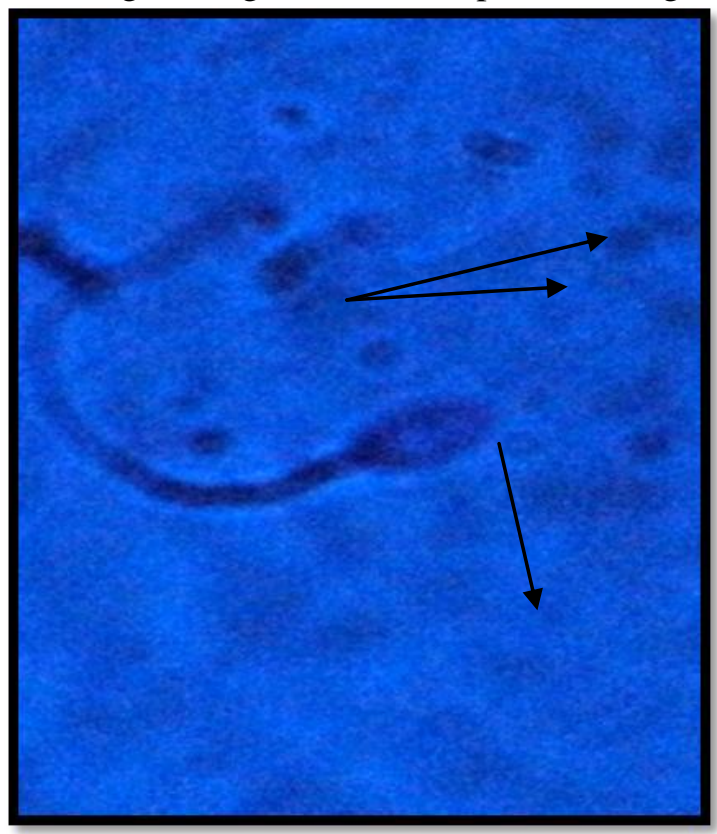

Fig:7 narrow with bent mid piece(O.I)

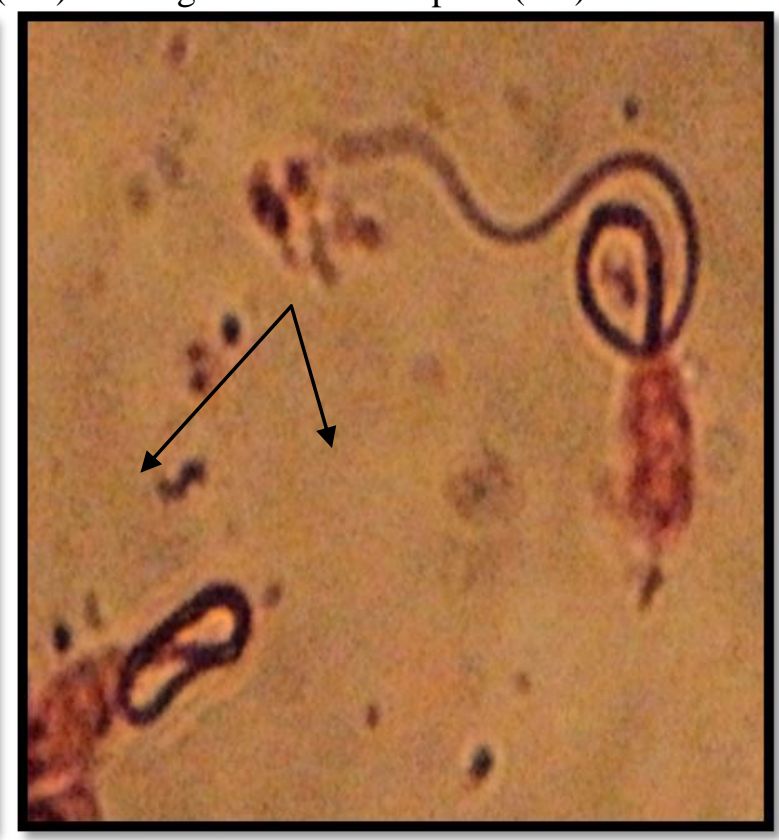

Fig:8 short with thickness mid piece, UV light (O.I)

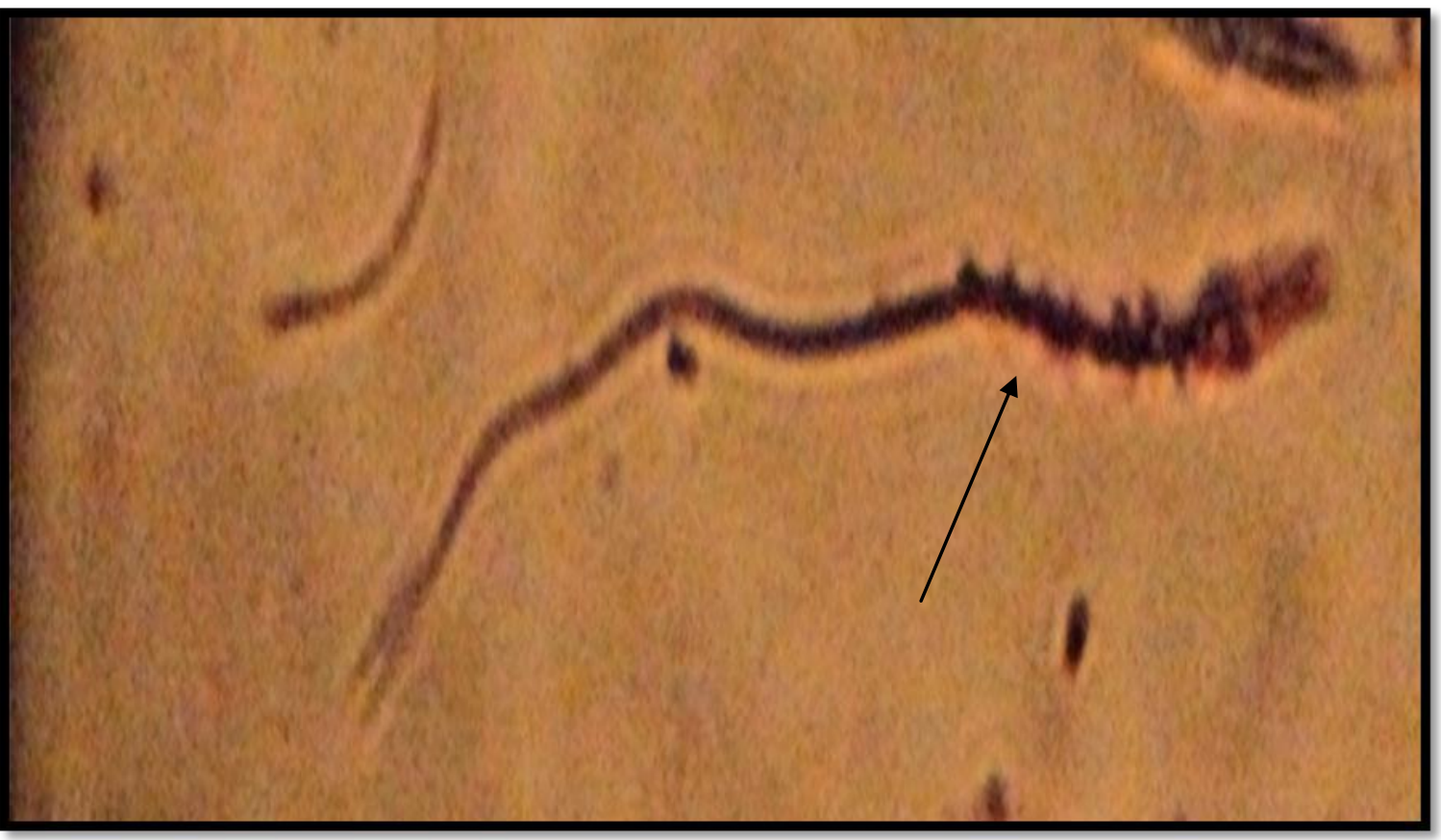

Fig:9 irregular mid piece .(O,I) 
Table 1 : show the types of defect in the mid pieces of sperms

\begin{tabular}{|c|c|c|c|c|c|c|}
\hline no & Interrupted & narrow & thickness & irregular & short & absent \\
\hline 1 & + & & + & + & & \\
\hline 2 & & & & + & & + \\
\hline 3 & & & & & & \\
\hline 4 & & & + & & + & \\
\hline 5 & & & + & + & & \\
\hline 6 & & & & & & \\
\hline 7 & & & & & + & \\
\hline 8 & + & + & + & & + & \\
\hline 9 & + & & & & & \\
\hline 10 & & & & & & \\
\hline 11 & & & & + & + & \\
\hline 12 & & & & + & & \\
\hline 13 & & & & & + & \\
\hline 14 & & + & + & & & \\
\hline 15 & + & & & & & \\
\hline 16 & & & + & & & \\
\hline 17 & & & + & & & \\
\hline 18 & & & & & & + \\
\hline 19 & & & + & & & \\
\hline 20 & & + & + & & & \\
\hline 21 & & & & + & & \\
\hline 22 & + & & & + & + & \\
\hline 23 & & & + & & & \\
\hline 24 & & + & & + & + & \\
\hline
\end{tabular}

\section{Discussion}

Low motility of sperms $(20 \%-40 \%)$ may be due to the mitochondrial sheath defect, or immature samples . MtDNA content may serve as a useful indicator of sperm quality and that mtDNA depletion may play an important role in the pathophysiology of some male infertility, a decrease in sperm mtDNA content was detected in patients with asthenospermia or with poor sperm motility $(<20 \%$ motility). (10).Although the mitochondrial sheath of spermatozoa clearly has a functional role, rather surprisingly there is little agreement about the relative importance of mitochondrial respiration ( as opposed to glycolysis ) for motility, or even for fertilization itself (11) Glycolysis is crucial for sperm functions (motility and fertilization) (12).Mid piece of sperms stained with ethiduim bromide in order to visualized mitochondrial sheath ( mitochondrial genome). Ethiduim bromide is a florescence reagent widely used in genomic analysis (13). Mitochondria , contain their own independent genome (DNA) and expression mechinary (14) Mitochondrial sheaths were visualized using the mitochondrion-specific vital dye MitoTracker green (15).The total number of sperm samples 24, were collected from tail of epididymis in rams, The defect in the mid piece of sperms, include 5 cases interrupted distribution of mitochondrial sheeth ,discontinues of mid piece staining because discontinues of mitochondrial 
sheeth, and 4 narrow mid piece, may be lose of mitochondrial sheeth . (16) Recorded, sperm with acute bendings at the level of the narrow midpieces. Another cases include 10 thickness, and 8 irregular .The defect consisted in a folding and coiling of the distal midpiece characterized by disorganization and irregularity of mitochondria surrounding the axial fiber bundle (17). Another 7 short mid piece observed in our study.(15) Mention , the Phase contrast and fluorescent microscopy of semen samples showed large numbers of spermatozoa with short, rigid, thick and irregular tails. The short of mid piece due to little of the number of helical mitochondrial sheeth, (18) Mention, mid- piece of tail with 7-13 helical mitochondria .Therfore this phenomena lead to variant of mid piece size, then the defect appeared in the short mid piece. Eventually, the absent of mid piece observed in 2 cases. In study of mid piece defect (16) recored mitochondria were either scarce or absent. This defect of mid piece lead to low activity due to low respiration . Mitochondrial respiration accounts for about $90 \%$ of cellular oxygen consumption (19) therefore any defect in mitochondrial genome lead to low metabolism and cell death pathways. The genome contains genes coding for 13 polypeptides involved in respiration and oxidative phosphorylation (20)

\section{References}

1.Ricki Lewis . Human Genetics, concept and application .( 2007 ). Seven edition :419.

2.Salisbury ,G.W. Van Demark, N .L .and Lodge.JR. (1978). Physiology of Reproduction and Artificial Insemination of Cattle . (Eds.). San Francisco . USA . 261.

3.Bayard T. Storey(2008)Mammalian sperm metabolism: oxygen and sugar, friend and foe Int. J. Dev. Biol. 52: 427-437.

4. Maureen M, Liliana T. Colombero, Lucinda L. Veeck, Zev Rosenwaks and Gianpiero, D. Palermo (1999) Sperm integrity is critical for normal mitotic division and early embryonic development Molecular Human Reproduction. 5, 9: 836-844.

5.St John, J.C., Sakkas, D. and Barrat, C.L.R. (2000) A role for mitochondrial DNA and sperm survival. J. Androl., 21, 189-199.

6.Alexeyev, M. F. LeDoux ,S.P.and Wilson,G.L.(2004)

Mitochondrial DNA and aging . Clinical Science .107,355-364.

7.Wiesner RJ, Ruegg JC, Morano I (1992). "Counting target molecules by exponential polymerase chain reaction, copy number of mitochondrial DNA in rat tissues". Biochim Biophys Acta. 183 ,2: 553-559.

8.May-Panloup,M-F. Chrétien, F. Savagner, C. Vasseur, M. Jean, Y. Malthièry,P. Reynier. (2010) Increased sperm mitochondrial DNA in male infertility. Human Reproduction. 550-556.

9.Sambrook , J ; Fritsch , E . F . and Maniatis . ( 1989 ) . Molecular cloning, $2^{\text {nd }}$ edition . Cold spring Harbor Laboratory Press , N.Y.

10. Shu-Huei, K. Hsiang-Tai, Chao. HwanWun, L.Tien-Lin, L.andYau-

Huei, Wei. (2004) Sperm mitochondrial DNA depletion in men with asthenospermia . Fertility and Sterility. 82, 1:66-73

11. Cummins, J.( 1998) Mitochondrial DNA in mammalian reproduction. Reviews of reproduction . 3, 172- 182

12. G Kamp, H Schmidt, H Stypa, S Feiden, C Mahling and G Wegener (2007) Regulatory properties of 6phosphofructokinase and control of glycolysis in boar spermatozoa . Reproduction .133: 29-40.

13. Kinji Mlura (1998) DNA detection with EtBr, Application Note ,No.3 sginfo@tokyo.fujifilm.co.jp 
14. Marcella,A.Domenico,C.Carmela,G.Gi orgio,G.Flavio,L.Sabino,L.Monic a,S. Graziano,P. and Cecilia, S. (2002). MitNuc: adatabase of nuclear genes coding for mitochondrial proteins .Nucleic Acids Research . 30, 1 : 172-173.

15. Rawel, V.Y. Galaverna, G.D. Acosta, A.A. Chemes, H.E.and Brugo Olmedo, S. ( 2010 ) Incidence of tail structure distortions associated with dysplasia of the fibrous sheath in human spermatozoa Human Reproduction .879-886

16. Rawe VY, Hermes R, Nodar FN, Fiszbajn G, Chemes HE.( 2007). Results of intracytoplasmic sperm injection in two infertile patients with abnormal organization of sperm mitochondrial sheaths and severe asthenoteratozoospermia. Fertil Steril Sep;88(3):649-53.
17. Andersen Berg K, Filseth O, Engeland E (1996)A sperm midpiece defect in a Hereford bull with variable semen quality and freezability. Acta vet scand .37(3):367-73.

18. Ropstorf P, Healy JM, Riedel F, Sitnikova TY( 2002)Comparative sperm ultastructure of baikalian endemic prosobranch gastropods. J Mollusca stud68 (2):111-126.

19. Walter De Gruyter .(3003) Mitochondrial oxidative stress and mitochondrial DNA Clin Chem Lab Med 41 (10) 12811288 .

20. Ji-Gang, D. Xia, L. Jia-Xin, M. GuoQiang, Z. Hong, W. (2005)Mitochondrial DNA sequence analysis of two mouse hepatocarcinoma cell lines World J Gastroenterol $14 ; 11,2: 264-267$

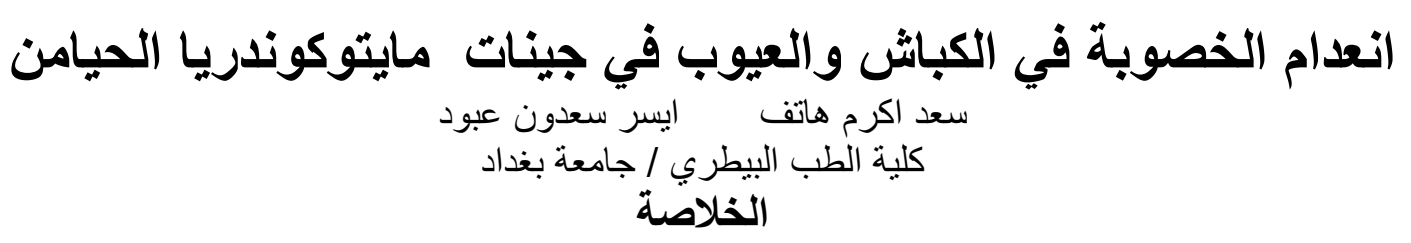

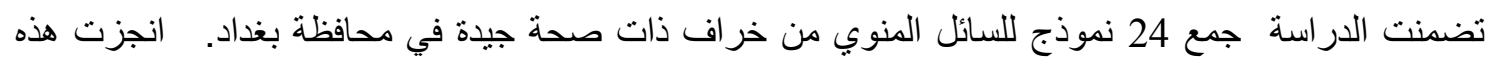

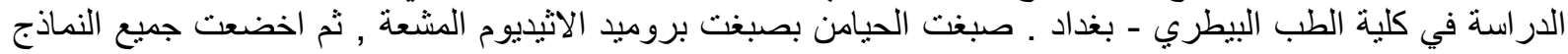

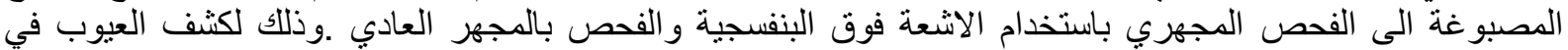

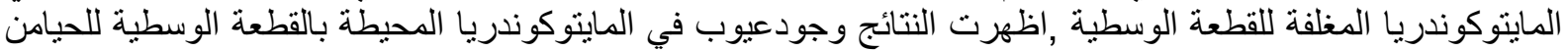

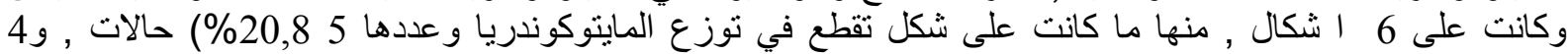

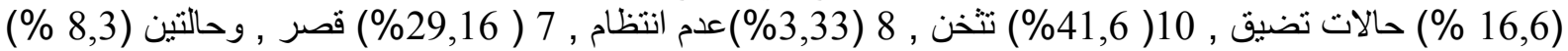

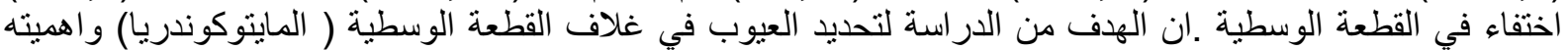

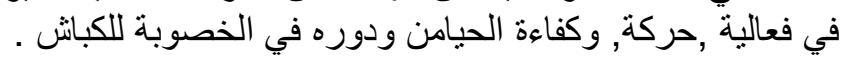

